\title{
METHOD FOR USING SOLAR ENERGY TO GENERATE CLEAN ENERGY BASED ON VACUUM-ATMOSPHERIC POWER AMPLIFICATION TECHNOLOGY
}

\author{
Igor Dubynskiy, \\ Academician, Leading Researcher, \\ G. V. Kurdyumov Institute for Metal Physics of the N.A.S. of Ukraine, Kiev, Ukraine
}

Andrey Dubinsky, Corr-member, Researcher Ukrainian Academy of Sciences, Kiev, Ukraine

DOI: https://doi.org/10.31435/rsglobal_ws/31052020/7071

\section{ARTICLE INFO}

Received: 25 March 2020

Accepted: 12 May 2020

Published: 31 May 2020

\section{KEYWORDS}

clean energy, atmospheric energy, solar energy, gravity, vacuum technologies.

\begin{abstract}
New method of converting solar energy into useful work and generating clean energy based on the technology of vacuum-atmospheric power amplification has been developed. As an energy source this technology uses an external supply of potential energy of the atmosphere in the Earth's gravitational field, which exists everywhere and becomes useful when it is converted into the desired form for use.

In this case, clean energy is generated in the same way as in hydroelectric power plants. The main advantage of the proposed method is that the devices in which it is used generate energy stably, regardless of the time of day, weather and location. Clean energy absorbed from the atmosphere is independently generated with any necessary design capacity for a specific consumer in the desired location without the use of organic fuel and main power grids. The article presents a theoretical justification of the method of generating clean energy based on the VAPA technology. Examples of using this method in various devices are given.
\end{abstract}

Citation: Igor Dubynskiy, Andrey Dubinsky. (2020) Method for Using Solar Energy to Generate Clean Energy Based on Vacuum-Atmospheric Power Amplification Technology. World Science. 5(57), Vol.1. doi: 10.31435/rsglobal_ws/31052020/7071

Copyright: (C) 2020 Igor Dubynskiy, Andrey Dubinsky. This is an open-access article distributed under the terms of the Creative Commons Attribution License (CC BY). The use, distribution or reproduction in other forums is permitted, provided the original author(s) or licensor are credited and that the original publication in this journal is cited, in accordance with accepted academic practice. No use, distribution or reproduction is permitted which does not comply with these terms.

Introduction. The technology of vacuum-atmospheric power amplification (hereinafter VAPA) is based on the use of the potential energy of the atmosphere in the gravitational field of the Earth. Until an apple stimulated Newton to create the Law of universal gravitation, no one thought about why a body without support necessarily falls down. When our $400 \mathrm{~kg}$ vacuum pump suddenly rose and began to "float" in the air relative to the support, we decided to investigate this phenomenon and find a practical application for it. Modern vacuum technologies have achieved significant development, which makes it possible to apply them in a fundamentally new direction - the generation of renewable clean energy by atmospheric-vacuum converters. This technology allows you to create environmentally friendly vacuum-atmospheric engines (hereinafter - VAE) with an external inclusive supply of nonthermal energy from a natural inexhaustible source - the potential energy of the atmosphere.

It is known that the surface of our planet constantly receives an average of $1.36 \mathrm{~kW} / \mathrm{m}^{2}$ of solar energy, a significant part of which is accumulated by the atmosphere. The atmosphere, as an open system, is a natural substance that stores solar energy, thereby maintaining the pressure and temperature gradient of the atmospheric layer in the Earth's gravitational field. [1;2]

Currently, the kinetic energy of the atmosphere is used for generating clean energy in the form of local winds at wind farms, which have a number of significant disadvantages. These include: 
instability of the air flow, the huge size of turbines for generating relatively large capacities, stationary binding to the terrain. The VAE turbine is 750 times more efficient than a three-blade wind turbine, which can provide an average output of no more than $400 \mathrm{~W} / \mathrm{m}^{2}$, and a rotary VAE of similar power can consistently provide a performance of more than $300 \mathrm{~kW} / \mathrm{m}^{2}$ from the blade surface when the turbine rotates at $120 \mathrm{rpm}(2 \mathrm{rpm})$, at any time of the day, regardless of the weather and location.

The purpose of this article is a theoretical justification of this method of generating clean energy and the possibility of practical use of the potential energy of the atmosphere as an external source of non-thermal energy for the production of useful work. The article describes the operation of various devices that use the potential energy of an atmospheric column, which is based on a work surface that separates media with different densities. In devices using this technology, work is performed on a vacuum-atmospheric continuous cycle with an external supply of non-thermal energy without the "dirty" technology of exclusive thermodynamic expansion of the working body, which is currently used.

\section{Interaction of solids on the boundary of two medium with different density in a gravitational field.}

On the border of two medium: water - atmosphere, in accordance with the law of Archimedes, it is possible to move loads in the gravitational field with a mass of $100-500$ thousand tons that are in suspension state in water due to the compensating hydrostatic (Archimedean) lifting force. Using the lifting force of the atmosphere is more difficult. The air density is $1.29 * 10^{-3} \mathrm{~g} / \mathrm{cm}^{3}$, the lifting force acting on a ball with a volume of $1 \mathrm{~m}^{3}$ that is filled with hydrogen with a density of $9 * 10^{-5} \mathrm{~g} / \mathrm{cm}^{3}$ will be approximately $12 \mathrm{~N}$. If a vacuum is created inside the shell of the ball, then the lifting power of the ball without taking into account the weight of the shell is $13 \mathrm{~N}$.

As a special case of Archimedes ' law, we can consider a variant when the lifting force acting on the movable surface of the shell, which is located on the border of two media - the atmosphere and the vacuum, increases by several orders and can reach a value of $101,325 \mathrm{~N}$ per $\mathrm{m}^{2}$.

Let's assume that the atmosphere at the Earth's surface is uniform, and the density is constant $\rho=$ const and the continuity condition is fulfilled $\frac{\partial \rho}{\partial t}=0$. Then the atmospheric pressure forces (hereinafter referred to as APF) act on the body as the volume forces $F_{a}$ for hydrostatic pressure in accordance with Euler's equations. In our case, for atmospheric pressure $P_{\mathrm{a}}$, can be represented as:

$$
\rho F_{x}=\frac{\partial P a}{\partial x} ; \rho F_{y}=\frac{\partial P a}{\partial y} ; \rho F_{z}=\frac{\partial P a}{\partial z} .
$$

Or in vector form:

$$
\rho F=\operatorname{grad} P_{\mathrm{a}}
$$

when $\rho=$ const, in the absence of volumetric forces $\operatorname{grad} P_{\mathrm{a}}=0$, the atmospheric pressure on all sides of the body is the same (Pascal's law). In the field of gravitational forces, the only force of gravity $F_{t}=m g$ acts. In order to transfer a body to a suspended state in the atmosphere, it is necessary to have a compensating force $F_{a}=F_{k s}$ (hereinafter referred to as CFAP) opposite to $F_{t}$, which can be done under certain conditions. [3]

Consider these conditions. The atmosphere, as a natural storage of solar energy, is an open system in which this process is implemented. The potential energy of a column of atmosphere with height $h$ that falls on a surface with an area of $S_{\text {eff, }}$ is equal to:

$$
E_{p}=S_{e f f} g \int_{0}^{h} \rho(h) h d h=S_{t f f} g \rho_{0} \frac{1}{\lambda^{2}}[1-\exp (-\lambda h)(\lambda h+1)]
$$

in this case, a barometric formula is used for the density of air in the atmosphere, where, $\rho(h)=\rho_{0} \exp (-\mu h), \mu=M g / R T, M$ is the molecular weight of the gas, $R$ is the gas constant, $\rho_{0}$ is the density of air at altitude $h=0, T$ is the absolute temperature.

If we assume that at the Earth's surface the atmosphere is uniform and the density is constant $\rho_{0}=$ const, then the potential energy of the column of atmosphere that rests on the surface of the area $S_{\text {eff }}$, can be represented as:

$$
E_{p}=S_{\text {eff }} g \rho_{0} h^{2} / 2[\mathrm{~J}]
$$


This formula gives an expression for the natural potential energy of the atmosphere in the field of gravitational forces created by the energy of the Sun. It is used in the VAPA technology as an inclusive source of non-thermal energy for the production of useful work.

The VAPA technology uses the energy of a non-closed system, which continuously receives solar energy, so according to the Second principle of thermodynamics, the operation of devices produced by this method does not contradict the Law of conservation of energy.

Therefore, devices for generating renewable clean energy, which are based on the VAPA technology, do not belong to perpetual motion engines, they can work, as well as hydroelectric power plants, in continuous mode without supplying organic fuel.

The principle of converting the potential energy of the atmosphere in the VAPA technology is similar to converting the energy $E=m g h$ of a falling stream (column) of water into electricity in hydroelectric power plants. Formula (4) can be represented as: $E_{p}=\alpha m_{a} g h_{l}$, where $m_{a}=\rho_{0} S_{\text {eff }} h$, is the mass of the atmospheric column with height $h$, which rests on a surface with area $S_{\text {eff, }}, h_{1}$ - the height (distance)at which the atmospheric column descends, $\alpha=\left(h-h_{1}\right) 2-$ a constant. It follows that the pressure force of the atmospheric column on the surface $S_{\text {eff }}$, is equal to $F_{a}=m_{a} g$.

Let us consider, as a special case of Pascal's law, the possibility of compensating the gravitational force $F_{t}$ due to the oppositely directed force of atmospheric pressure $-F_{k s}$ and its use for performing useful mechanical work on a closed vacuum-atmospheric cycle (Fig. 1.). [4]

Let's assume that we have a sealed cavity 1 in the form of a cylinder whose side surface is elastic, for example, in the form of a bellows 3, the ends of which have an area of $S_{\text {eff }}=1 \mathrm{~m}^{2}$. One end of the bellows is rigidly fixed to the support surface 5 . The body 7 is attached to the lower end 2 , which has the ability to move freely up/down relative to the vertical supports 4 .

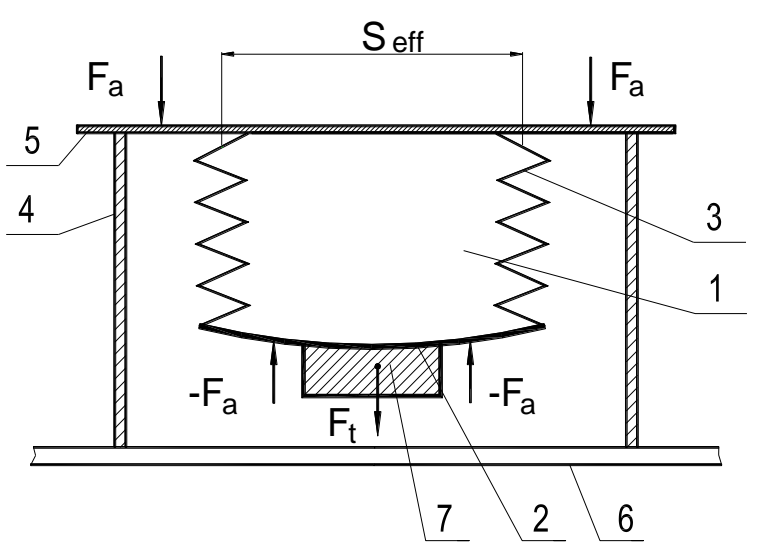

Fig. 1. Interaction of forces CFAP $F_{k s}=-F_{a}$ and force of gravity $F_{t}$

The atmospheric pressure on a surface area of $S_{\text {eff }}=1 \mathrm{~m}^{2}$ equals to $101325 \mathrm{~Pa}$ or $101 \mathrm{kN} / \mathrm{m}^{2}$, and force $F_{a}$, with which atmospheric pressure $P_{a}$ acts on a surface area of $1 \mathrm{~m}^{2}$ equals to:

$$
F_{a}=F_{k s}=P_{a} S=101325 P a * 1 m^{2}=101325[\mathrm{~N}]
$$

If air is pumped out of the cavity 1 , then, in accordance with Pascal's law, the compensating vertical component $-F_{a}=F_{k s}$, which acts on the surface of the end of the bellows 2 , is also equal to $101 \mathrm{kN} / \mathrm{m}^{2}$, This force, which will later be called the Pascal force, can lift a load 7 weighing 10,000 kg into a suspended (levitating) state relative to this support, if it is attached to the movable end face/platform of the vacuum cavity 2, which has an area of $1 \mathrm{~m}^{2}$ (hereinafter - PP). In this case, the body weight is redistributed to the support $(4,5)$.

CFAP is always normal at any point in the separation shell of two media with different densities, which is supported by an atmospheric column.

The Pascal force $F_{a}$ inclusively acts in any direction in the atmosphere on a separation shell with an effective area of $S_{\text {eff }}$, which is located between two media-the atmosphere and a vacuum or between a liquid and a vacuum.

In General this physical phenomenon can be formulated:

The Pascal force equal to the weight of the atmospheric / liquid column, which rests on the opposite surface of this vacuum cavity with area S, fixedly mounted on the support, is inclusively 
acting on the moving surface with area $S$ of the vacuum cavity with an elastic side shell that is immersed in the atmosphere or liquid.

On the basis of f. 5, it is obvious that the compensating force acting on the body, which is located on the PP vacuum cavity relative to the support, is the lifting force of Pascal $F_{k s}$, which significantly exceeds the pushing force of Archimedes $F_{a r h}$.

The total pushing force $F_{a r h}$ on the submerged body is equal to:

$$
F_{\text {arh }}=\rho_{t} g V_{t}
$$

where $\rho_{t}-$ is the density of the body, $V_{t}-$ is the volume of the body, $g$ - is the acceleration of gravity. [5]

As shown above, for a ball with a volume of $1 \mathrm{~m}^{3}$, from which the atmosphere is pumped out, the buoyancy force will be: $F_{a r h}=13 \mathrm{~N}$

The potential Pascal force with which the atmospheric column presses on the PP of the vacuum cavity on a support that has an area of $S_{\text {eff }}=1 \mathrm{~m}^{2}$, based on f. 4; 5 is equal to:

$$
F_{k s}=\rho_{0} g V_{a}=P_{a} S_{\text {eff }}=101325[N]
$$

where $\rho_{0}-$ is the density of the atmosphere, $V_{a}-$ is the volume of the atmospheric column above the PP with $S_{\text {eff. }}$.

To create such a buoyancy force in the atmosphere, Archimedes will need an airship with a volume of $8436 \mathrm{~m}^{3}$ filled with hydrogen.

It is obvious that a sealed cavity 1 in the form of a cylinder, in which the side surface is elastic, for example, in the form of a bellows 3 (Fig. 1), can provide a transition to a levitating state in the atmosphere relative to the support of physical bodies of almost any weight. This allows you to create simple vibration-isolating systems based on VAPA technology - vacuum-atmospheric stabilizers of objects relative to the horizon. [6]

The main provisions of the theory of VAPA technology

Obviously, under the influence of Pascal's force, the movable end surface of the vacuum cavity can produce inclusive useful work in a closed vacuum-atmospheric cycle due to the potential energy of the atmosphere. Useful work is done due to the interaction of gravity and atmospheric pressure and can become the basis for the development of a fundamentally new technology for generating clean energy.

Based on the above, it is possible to formulate the following provisions that determine the operation of the VAPA technology:

1. The mobile surface of a hermetically closed vacuum cavity, rigidly fixed to a support located in the external environment - atmosphere/liquid, is constantly affected by the force of atmospheric pressure - the Pascal force from this external environment. The Pascal force acting on the movable end surface is always directed in the opposite direction to the stationary end surface of the vacuum cavity with a flexible side shell. This force is directly proportional to the effective area of the stationary and mobile surface and is equal to the pressure difference between the external environment and the vacuum cavity.

2. The body may be in a suspended (levitating) state in the atmosphere (liquid) if it is attached to a hermetically sealed vacuum cavity with an elastic lateral surface fixed to the support in the external environment - the atmosphere (liquid). In this case, the force of gravity is compensated by an equal Pascal force.

3. The movable surface of the hermetically sealed vacuum cavity, which is on the support and divides two media with different density: the atmosphere-vacuum or liquid-vacuum, can produce useful work. This work is done from an external source of non-thermal energy, directly proportional to the volume of the vacuum cavity and the pressure difference in the cavity and the external environment.

This implies:

Consequence 1. The Pascal Force acting inclusively on the mobile surface of a hermetically closed vacuum cavity, which has a lateral elastic shell and a support point in the external environment, can compensate for the force of gravity and keep the body in a suspended (levitating) vibration-insulated state in the atmosphere. The value of the compensating force is determined by the specified effective area of the fixed support surface and the pressure in the vacuum cavity. 
Consequence 2. The movable end face of a hermetically sealed vacuum cavity, which has a point of support in the external environment, can continuously produce useful work under the action of Pascal's forces at the expense of the potential energy of the external medium in the atmosphere or liquid if the volume and pressure in the cavity continuously vary in a closed vacuum-atmospheric cycle.

Consequence 3. The energy that is spent to create a vacuum in the vacuum cavity in the vacuumatmospheric cycle can be less than the potential energy of the external medium - the atmosphere or liquid expended for work, which is produced by the movable surface of the vacuum cavity.

Consequence 4. Conversion of the potential energy of the atmosphere into free clean energy can occur, provided that the energy spent on creating a vacuum is less than the energy absorbed from the atmosphere to produce useful work. [7]

\section{Practical application of the VAPA technology}

It is practically possible to move a platform with a load under the action of a force $F_{a}$ at a distance $l$ relative to the support located on a solid underlying surface, if a cavity is formed between the platform and the support plane, in which a vacuum environment is artificially created (see Fig. 1.).

For example, a platform with an area of $S=1 \mathrm{~m}^{2}$ when passing a distance of $l=1 \mathrm{~m}$ under the influence of the force $F_{a}$ of the atmospheric column, which presses on the effective area of the outside of the platform, will produce work:

$$
A=F_{a} l=101 k N^{*} 1 m=101[k J
$$

and can provide $100 \mathrm{KW}$ of useful power per second.

$$
N=A / t=101[k W]
$$

It is obvious that based on the expression (9), the useful power is determined by the pumping speed of the volume of the vacuum cavity $V=S l$, at $P_{a}=$ const, while it is obvious that 1 liter of volume, due to the absorbed potential energy of the atmosphere, can generate $100 \mathrm{~W} / \mathrm{s}$ of useful power.

To return the platform to its original position, it is necessary to fill the vacuum cavity with atmospheric air in a natural way (by pressure) to compensate for the effect of the force $F_{a}$ of the atmospheric column on the outside of the platform. Thus, a two-stroke vacuum-atmospheric cycle can be obtained: "pump out - air inlet". If it is possible to cycle the platform at a distance of $\pm l$ with a frequency of $10 \mathrm{~Hz}(600 \mathrm{rpm})$, it can provide $1 \mathrm{MW}$ of power for mechanical work due to the absorbed potential energy of the atmosphere.

When converting the forward movement of the platform to the rotation of the shaft knees, the torque is defined as:

$$
M_{t}=F r=S P_{a} l / 2[N m]
$$

and the output power can be written as:

$$
N=n P_{a} S r[W]
$$

Where: $n$ - the number of shaft speed; $P_{a}-$ atmospheric pressure; $S$ - the effective area of the external surface of the platform; $r$ - the radius of the point of application of force.

This formula almost completely defines the main parameters of a power plant using the crank mechanism $\mathrm{CM}$, in which the absorbed potential energy of the atmosphere is used as an external source of non-thermal energy.

For example, at normal atmospheric pressure $P_{a}=1.013 * 10^{5} \mathrm{~Pa}$, the torque for the case in question will be:

$$
M_{t}=1,013 * 10^{5} \mathrm{~S} l / 2=50650[\mathrm{Nm}]
$$

and the output power at $\mathrm{n}=10$ will be:

$$
N=1,013 * 10^{5} n \mathrm{Sr}=500[\mathrm{~kW}]
$$

To almost create a power plant it is necessary to solve three problems: to find a way of converting potential energy into kinetic atmosphere; to create the ability to produce mechanical work; to create an engine that will be able to work through external source of non-thermal energy pressure of the atmosphere. The solution of these problems allows using a fundamentally new source of clean energy, the operation of which does not depend on the action of the wind, the flow of falling water, the 
time of day and location (except for the height above sea level). At the same time, this energy source can be created with any given useful output power from 3-15 kW to 1-10 MW or more, which will work in accordance with the laws of conservation and thermodynamics as hydroelectric and wind power station.

The creation of a vacuum-atmospheric engine VAE and a device for generating electric energy, which uses the potential energy of the atmosphere as an external source of non-thermal energy, has been solved by us in several variants and is protected by patents of Ukraine and the EAPO [8;9], as well as a US patent [10].

Fig. 2. shows a scheme of a device that can perform mechanical work due to the potential energy of the external environment, while the vacuum system provides the necessary speed for pumping the working fluid (gas) from the vacuum cavity in a closed cycle without escaping into the atmosphere.

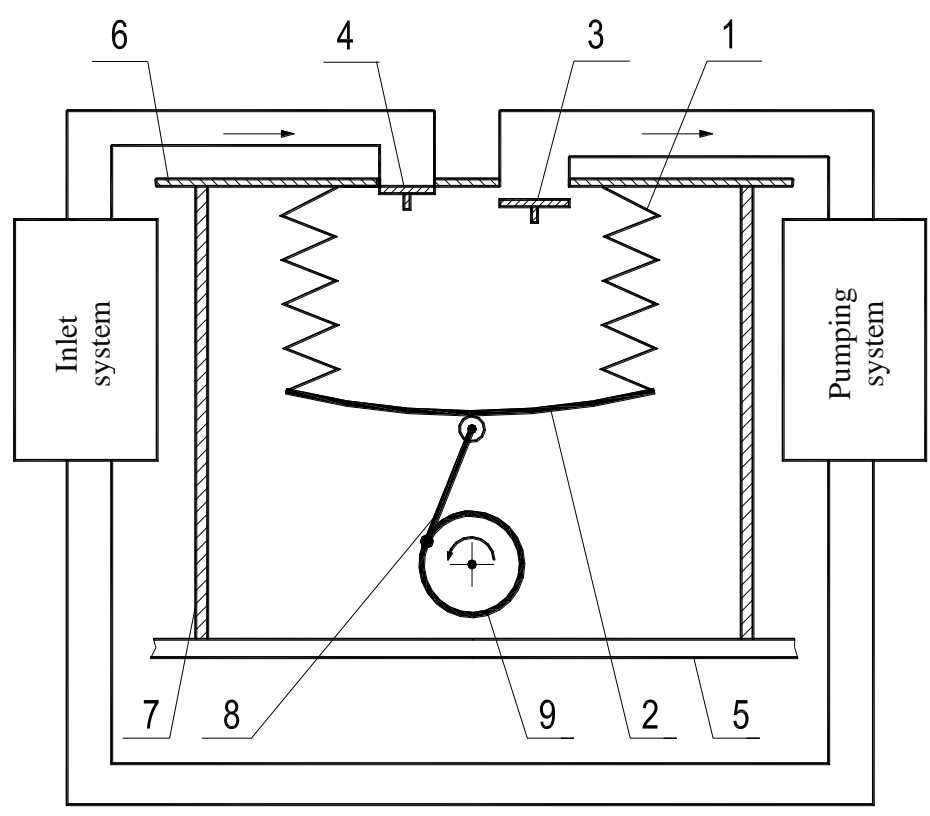

Fig. 2.

Where: 1 - elastic lateral surface (bellows) of the vacuum cavity; 2 - a moving surface (an end face of a bellows); 3; 4 - evacuation / inlet valves; 5 - underlying surface; 6 - supporting surface; 7 - stand support; 8 - connecting rod; 9 - a cranked shaft with a flywheel.

Fig. 3. shows a variant of the experimental sample with one working bellows. The device operates in a push-pule vacuum-atmospheric cycle mode. Shaft rotation is controlled by Hall sensors.

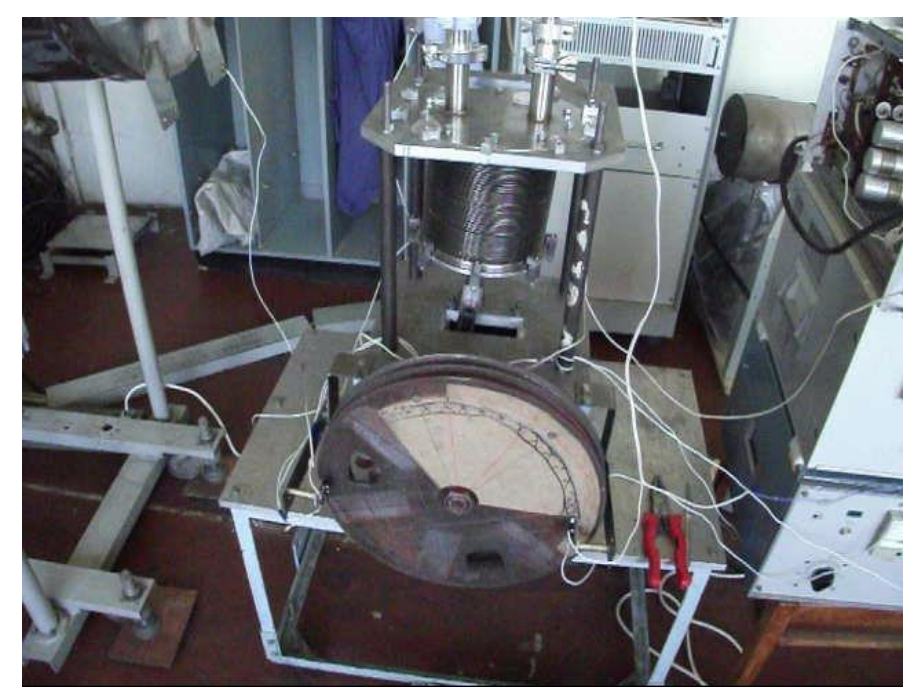

Fig. 3. Variant of the experimental sample with one working bellows 
Thus, the power part of the plant that generates electricity from an external source of clean energythe absorbed potential energy of the atmosphere, works in isolation and independently relative to the vacuum pumping that consume electricity, creating an artificial vacuum in the cavity. A positive balance between generated and consumed energy and will be the useful clean energy that is generated by the device. The choice of an effective vacuum system will determine power gain $G p$ of the concrete device. [8]

As an example, for calculating the $G p$ of the device, we take two versions of the standard oilfree dry vacuum system with a Roots pump (main) and a screw auxiliary pump with parameters: a. productivity $300 \mathrm{l} / \mathrm{s}$; power consumption $11 \mathrm{~kW}$;

b. productivity $2500 \mathrm{l} / \mathrm{s}$; power consumption $51 \mathrm{~kW}$.

The work that this vacuum system performs to pump out a vacuum cavity with a volume of $V_{0}=1\left[\mathrm{~m}^{3}\right]$ at a speed of $v\left[\mathrm{~m}^{3} / \mathrm{s}\right]$, at a power consumption of $N[\mathrm{~W}]$ is defined as:

$$
A=N V_{0} / v[\mathrm{~J}]
$$

For the example «a» with parameters $v=0,3 \mathrm{~m}^{3} / \mathrm{s}, N=11 * 10^{3} \mathrm{~W}$, the work $A_{l}$ will be:

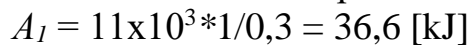

For the example «b» with parameters $v=2,5 \mathrm{~m}^{3} / \mathrm{s}, N=51 * 10^{3} \mathrm{~W}$, the work $A_{2}$ will be: $A_{2}=51,5 * 10^{3} * 1 / 2,5=20,6[\mathrm{~kJ}]$

Thus, $36.6 \mathrm{~kJ}$ and $20.6 \mathrm{~kJ}$, respectively, were spent on pumping out at a given speed of the vacuum cavity with a volume of $1 \mathrm{~m}^{3}$ by the vacuum system. It is very important that the pumping is carried out due to work, for which the energy of a closed system is used from generating devices, for example, through centralized power networks or from autonomous diesel power plants. However, the working mechanism of the power part of the installation with the volume of the vacuum cavity $V_{0}=1 \mathrm{~m}^{3}$, produces stable effective work $A_{\text {ew }}$ directly from the energy source of the open system - the potential energy of the atmosphere, which is a continuous natural storage of solar energy. This work is performed stably with the support in the vacuum cavity of the pressure $P_{0} \leq P_{a t}^{-2}$ equals to $A_{e w}=101 \mathrm{~J}$.

It follows that the power gain $\mathrm{Gp}$ of the device depends on the parameters of the vacuum system and in this particular case will be equal to:

For example, «a» $G p=A_{e w} / A_{l}=101 / 36.6=2.76$,

For example, «b» $G p=A_{e w} / A_{2}=101 / 20.6=4.9$.

In a real device, $G p$ can be twice as high, as in the above calculations, it was not taken into account that during the working stroke the volume $V_{0}$ of the vacuum cavity is a variable.

One of the applications of $F_{a}$ in a closed vacuum-atmospheric cycle can be carried out in a device where two PPs in the form of movable cylinders rigidly connected through a crank form a power pair and move back and forth along a push-pull cycle. Fig. 4. shows a diagram of such a device using a cylinderpiston group and a crankshaft. In this case, each clock cycle of the device is operational. If one cylinder carries out a working stroke, pumping out of its cavity occurs and Pascal force acts on its end face, then the second cylinder idles, the atmosphere is naturally poured into the cavity, the pressure on both ends is the same and the Pascal force does not act. During the reverse stroke, the cavity of the second cylinder is pumped out, and it makes a working stroke, and the first cylinder idles, while the atmosphere is poured into its cavity in order to compensate (neutralize) the Pascal force. [9]

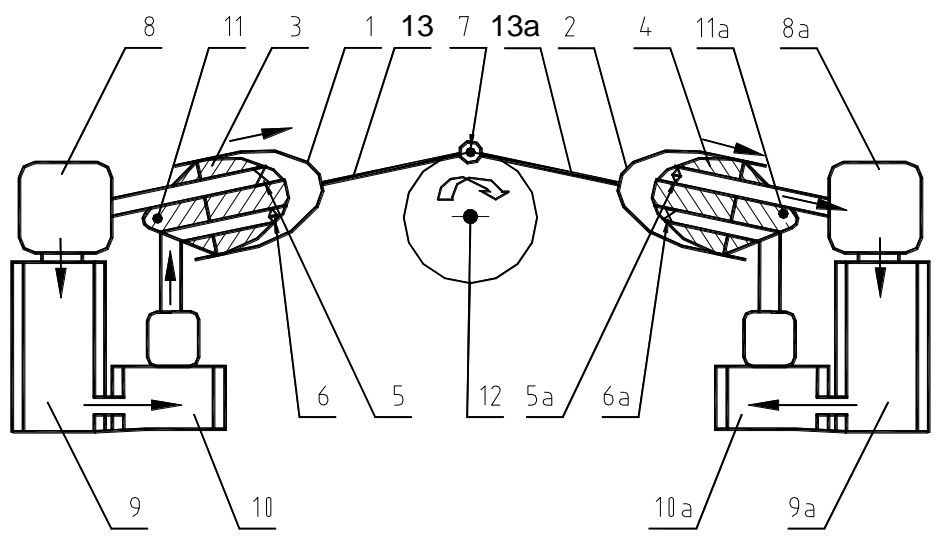

Fig. 4. Scheme of the device with movable cylinders 
Where: 1,2 - movable cylinders; 3,4 - pistons with the ability to swing on the axes $11,11 \mathrm{a} ; 5,5 \mathrm{a}$, $6,6 \mathrm{a}$ - inlet / exhaust valves; 7 - the axis of the crank; 8, 8a, 9, 9a- pumping system; 10, 10a - the inlet system; 12 - an axis of the crankshaft; 13, 13a - rods connecting movable cylinders with the eccentric axis of the crank.

Due to the fact that $F_{a}$ during the working stroke is constant in absolute value, the use of the crank mechanism, on which up to $50 \%$ of the power is lost, is not rational in high-power generation devices. Therefore, in this method of generating large powers, it is optimal to use a VAT vacuumatmospheric turbine, which can operate on the basis of VAPA technology as part of a vacuumatmospheric rotary power amplifier (hereinafter - VARPA).

\section{Principle of VARPA.}

As it was shown above, so that the Pascal force $F_{a}$ acts on the moving surface in a closed cycle, it is necessary to create a pressure difference in the vacuum cavities that this surface separates. To ensure the movement of the surface that separates the vacuum cavities, it is necessary that the volumes of these vacuum cavities simultaneously increase / decrease (see Fig. 4.).

This vacuum-atmospheric cycle of supplying the absorbed potential energy of the atmosphere to obtain mechanical work in one housing is implemented in a vacuum-atmospheric turbine (hereinafter - VAT), which is shown in Fig. 5. [10].

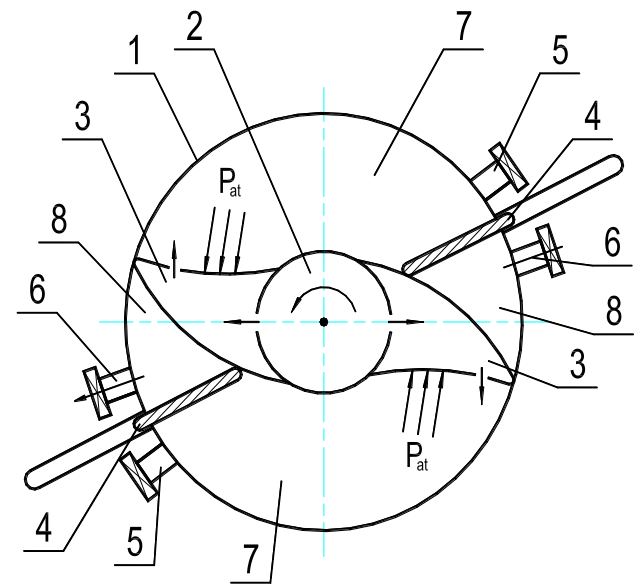

Fig. 5. VAT radial section

VAT contains a cylindrical housing 1, in which the rotor 2 is located, mounted in the housing of the device. The rotor is equipped with at least two streamlined blades 3 , the ends of which are in contact with the inner surface of the housing with the possibility of vacuum tight sliding on this surface. Movable plates / dampers 4 - are built into the casing. They are installed diametrically opposite in the VAT casing and divide its volume into two equal vacuum cavities formed by the outer surface of the rotor with blades and the inner surface of the casing. Atmosphere is inflated and evacuated through valves $5 ; 6$. The dampers provide separation of the vacuum cavities $7 ; 8$, they touch their ends with the outer surface of the rotor and the blades with the ability to slide vacuum tightly on this surface. As the blades rotate, which are affected by atmospheric pressure, the volumes of the vacuum cavities simultaneously increase / decrease. To ensure continuous atmospheric inlet into the constantly expanding cavity 7 , the rotor shaft and rotor blades are made hollow. On the surfaces of the rotor blades there are nozzles of the "Laval nozzle" type (shown by arrows) through which the atmosphere enters the cavity 7. At the same time, the decreasing volumes of the cavities 8 are continuously evacuated, in which the pressure $P_{0} \ll P_{a}$ is maintained by the vacuum system, which ensures a given pressure difference.

The rotation of the rotor is ensured by the direct influence of the Pascal force $F_{a}$ on the surface of the blades in the cavities 7 while evacuating the atmosphere from the cavities 8 . The blades are diametrically opposed relative to the rotor, which makes it possible to perform mechanical work with constant torque. On the rotor there can be more opposing blades more, which is determined by the design features of the VARPA to obtain the necessary parameters of the VAT. [11]

$V A T$ is much more efficient than a three-bladed wind turbine, which can provide an average output of no more than $400 \mathrm{~W} / \mathrm{m}^{2}$ from the flooded area, and the VARPA rotary amplifier provides 
continuously stable energy generation, for example, more than $300 \mathrm{~kW} / \mathrm{m}^{2}$ from the surface of the blade when the turbine rotates only at $120 \mathrm{rpm} / \mathrm{min}(2 \mathrm{rpm})$, at any time of the day, regardless of weather and location.

As an autonomous clean energy generator, VARPA can compete in all respects and completely replace diesel power plants, for example, in the range of capacities of $0.1-3 \mathrm{MW}$, which are in demand on the market mainly as autonomous standby sources of electricity. As a rule, they consume fossil fuels up to 200-400 liters / hour and cannot work on an ongoing basis. VARPA practically does not consume fuel and can generate electricity in constant mode for an unlimited time. Savings in fuel costs alone can be up to $\$ 3,000,000$ per year per installation.

The dimensions of the VARPA with a design reserve capacity of about 2 MW will be approximately the same with diesel power plants of the same power, and the weight, even taking into account the vacuum system, will be significantly less, so VARPA can be performed in a mobile version, for example, in a standard container.

2. Calculation of the delivered power of the VARPA device for 2 MW with the following parameters:

- the diameter of the rotor $\mathrm{D}_{1}=0.3 \mathrm{~m}$;

- inner diameter of the housing $\mathrm{D}_{2}=1.3 \mathrm{~m}$;

- the length of the blade along the axis of symmetry of the rotor $\mathrm{h}=1 \mathrm{~m}$;

- the length of the rotor with 4 blades $2.5 \mathrm{~m}$;

the total surface area of the four rotor blades will be $S=2 \mathrm{~m}^{2}$.

Substituting these parameters in the calculated formula of the device's power, we get the power output of the device at $120 \mathrm{rpm} / \mathrm{min}(2 \mathrm{rpm})$ :

$$
N=\pi / 2 P_{a} S n=3,14 / 4 * 101300 * 2 * 2=636[\mathrm{~kW}]
$$

If, according to the patent, we increase the surface area of the four blades in two chambers of the rotor to $S=5 \mathrm{~m}^{2},\left(\mathrm{D}_{2}=2.3 \mathrm{~m} ; \mathrm{h}=1.6 \mathrm{~m} ; \mathrm{D}_{\mathrm{av}}=1.3 \mathrm{~m}\right)$, then the power output of the two-chamber device at $180 \mathrm{rpm} / \mathrm{min}(3 \mathrm{rpm})$ increases to $2.38 \mathrm{MW}$. At the same time, the rotor length of the power unit increases to 3.5 meters, and the diameter of the camera bodies up to 2.5 meters. will be:

The torque on the power shaft of two chamber installation, in which the rotor has four blades,

$$
M=4 F r=2 F D_{a w}=101300 * 1,3 * 2=263380[\mathrm{Hm}]
$$

Such a torque ensures the stability of rotation of the rotor of the generator at even the maximum variable load.

To obtain the required pressure difference, it is necessary to ensure pumping of the working chambers and constantly maintaining a pressure in the pumped parts of the vacuum chambers of about 100-10,000 Pa. Modern dry vacuum pumps of serial production provide the necessary speeds for individual pumping of the vacuum cavities of the working chambers.

The optimal use of VARPA is possible as a powerful autonomous offshore source of clean electricity, in trucks, trunk locomotives and marine power units.

Due to the fact that the torque does not depend on the rotor speed and there are practically no thermal and mechanical losses, the VARPA power plant with these parameters can provide the required speed of a vessel with a sufficiently large displacement without the use of fossil fuels.

For example, for a ship, in this case, the rotor rotates at an average speed of $180 \mathrm{rpm}$, therefore, a power shaft with a propeller can be connected directly to the rotor without loss of power in the transmission. The rotational speed and stop of the rotor are regulated by the electronic controller by changing the rate of inlet and exhaust of the atmosphere.

Obviously, with the use of VARPA in a marine power plant, we can obtain, ceteris paribus, fuel savings of about 10 times, which is very important for long-term autonomous navigation. At the same time, VARPA will provide minimal vibration and noise of the power unit. Similar fuel economy can be obtained by using VARPA in the power unit of the main locomotive and truck.

\section{Conclusions.}

1. The potential energy of the atmosphere is a natural unlimited source of non-thermal clean energy and can be used to obtain useful work. 
2. It is shown that modern vacuum pumping systems make it possible to create devices that provide a transition to a suspended state in the atmosphere relative to the support of physical bodies of almost any weight.

3. A method for converting solar energy into useful work is theoretically substantiated, on the basis of which a vacuum-atmospheric power amplification technology is developed - VAPA technology, as a new promising direction in renewable energy. VAPA technology can have a great advantage over other methods of generating clean energy, which use the energy of the Sun. It can be widely used for non-thermal autonomous generation of clean energy due to the absorbed potential energy of the atmosphere.

4. Based on the VAPA technology, it is possible to create compact, almost silent autonomous devices for generating clean energy of any power, operating on an implosive non-thermal vacuumatmospheric cycle, which can be effectively used in the energy sector and as part of environmentally friendly power units of vehicles.

\section{REFERENCES}

1. Van Mieghem, J. Atmospheric Energetics. / Transl. from English by Matveev L.T. L.: Gidrometeoizdat, 1977. Retrieved from: https://www.twirpx.com/file/1150939/

2. Борисенков Е.П. Климат и деятельность человека. Retrieved from https://www.koob.ru/borisenkov/climate

3. E. H. Brandt. Levitation in Physics. Science 243, 349 (1989) Retrieved from: https://science.sciencemag.org/content/243/4889/349/tab-article-info

4. Dubinskiy A. I., Dubinskiy I. N., EAPO Eurasian patent, application № 013312 В1“Устройство для подвеса и перемещения груза относительно опорной и подстилающей поверхностей” 2010 г.

5. Гидростатика. Retrieved from: https://firing-hydra.ru/index.php?request=full\&id=528

6. Dubinskiy A. I., Dubinskiy I. N., EAPO Eurasian patent, application № 026774 B1 «The method and the device for gyroscopic stabilization of lidar in horizontal plane» 2017.

7. Dubinskiy A. «Energy XXIst problems and prospects for their non-standart solutions in world practice». К.: Издательство УАН НВП ВИР, София, 2013.

8. Dubinskiy A. I., Dubinskiy I. N., EAPO Eurasian patent, application № 013312 B1 "Device for suspension and movement of load regarding the support and underground surfaces" 2010

9. Dubinskiy A. I., Dubinskiy I. N., Patent Ukraine, application № UA 89894 С2 «Устройство для получения механической работы от внешнего источника нетепловой энергии» 2010 г.

10. I. Dubinskiy, A. Dubinskiy Patent No.: US 10,125,609 B2; Date of Patent: Nov. 13,2018

11. I. Dubinskiy, A. Dubinskiy. (2018) "Vacuum-atmospheric rotor power amplifier for generation of clean energy”. International Academy Journal Web of Scholar. 11(29), November 2018. Retrieved from https://doi.org/10.31435/rsglobal_wos/30112018/6240 